\title{
The Genomics of Industrial Process through the Qualia of
} Markovian Behaviour

This paper was downloaded from TechRxiv (https://www.techrxiv.org).

\section{LICENSE}

CC BY 4.0

SUBMISSION DATE / POSTED DATE

01-02-2022 / 05-02-2022

\section{CITATION}

Danishvar, morad; Danishvar, Sebelan; Mousavi, Ali (2022): The Genomics of Industrial Process through the Qualia of Markovian Behaviour. TechRxiv. Preprint. https://doi.org/10.36227/techrxiv.19102523.v1

DOI 


\title{
The Genomics of Industrial Process through the Qualia of Markovian Behaviour
}

\author{
Morad Danishvar, Ali Mousavi, Senior Member of IEEE, Sebelan Danishvar
}

\begin{abstract}
A technique for registering and relating events that cause an observable and definable system state is proposed. Discrete events of system state transfer are expressed by event tracking and clustering in the form of contiguous quanta of data. This approach is capable of describing typical processes in industrial systems in a chain of codes that contain system input/output parameters. The constituent nodes of the Markovian Processes chain form a series akin to genes in the DNA, repeatable and predictable. The process genes are the quanta of information that aligns to represent a chain of activities (process). They describe the causal links between occurring events forming a pattern (pathway) that leads to a well-specified output (e.g., a product with a defect or otherwise). The creation of process genomics requires the knowledge of system observed or latent parameters (state) as well as the state change at specified time intervals (discretization).

The process genomics theory is tested in an industrial case study for quality assessment and control of glue dispensing in micro-semiconductor manufacturing. The resulting definitions of system state and interrelationship of control parameters contribute to the development of the process genes. The outcome of the gene alignment is the geometric interpretation of the glue droplets formation. A predicted or observed droplet within the production tolerance leads to a non-defective product.

The principle of creating production genomics is to find and rectify the defect causing genes or to disrupt the sequences that lead to producing defective products, leading to a Zero-Defect manufacturing process.
\end{abstract}

Index Terms - Real-time, Markovian Process, Prediction, Machine Learning, Manufacturing, Data-driven, Quality, Dispensing Technology

\section{INTRODUCTION}

$\mathrm{S}$ ystems equipped with sensory inputs should be able to recognize and predict temporal sequences of events. A sequence defines as an ordered series of events. Depending on the type of events forms sequences, discrete, continuous, or binary sequences are some examples of different kinds of sequences. In real-life applications, we face these types of sequences that can be categorized between the essential forms of sequences.

Sequential behaviour analysis is a key element of human reasoning, complex problem solving and decision-making. Especially, sequence learning is a major module of learning domains such as natural language processing, machine learning, adaptive control, temporal prediction, financial

The authors are with College of Engineering, Design and Physical Sciences, Brunel University Kingston Lane, UB8 3PH, UK (e-mail:

Morad.Danishvar@brunel.ac.uk, Ali.Mousavi@brunel.ac.uk;

Sebelan.Danishvar@brunel.ac.uk) engineering, genome sequencing, and so on [1]. The process of this temporal information is also one of the fundamental aspects of human intellectual ability and his desire to replicate this in industrial automation and computing.

A sequential phenomenon of events can be expressed in the form of an ordered or random list of symbols or numbers. The knowledge about the list of events can be enhanced by, for example, registering the time they occur (timestamp). Event sequencing is, therefore, registering as an observed phenomenon at specified time intervals. The reported sensory data in such time series can be compared with earlier data, and state changes or non-change be determined. Equipped with such preliminary data and logical inference, there is a possibility to go beyond, and that is event sequence prediction (ESP). ESPs consist of predicting the occurrence of the next symbol(s) in a sequence based on previously observed symbols. If such an occurrence has not been observed previously, then a new event is registered.

It is safe to declare that the logic and chain of reasoning (theorem) of the proposed Genomics of Industrial Process (GIP) is closely related to Temporal Sequence learning (TSL) terminology. Imitating the human brain, the GIP learning process exploits temporal sequence and its rationale to explain and project the state of systems in timespace.

Sequence prediction is classified as an application of sequential data and attempts to predict elements of a sequence based on the preceding elements in (1).

$\mathrm{S}_{\mathrm{i}}, \mathrm{S}_{\mathrm{i}+1}, \mathrm{~S}_{\mathrm{i}+2}, \mathrm{~S}_{\mathrm{j}} \rightarrow \mathrm{S}_{\mathrm{j}+1}$.

$\left(\mathrm{S}_{\mathrm{i}}, \mathrm{S}_{\mathrm{i}+1}, \mathrm{~S}_{\mathrm{i}+2}, \mathrm{~S}_{\mathrm{j}}\right.$, is given and $\left.1 \leq \mathrm{i} \leq \mathrm{j}<\infty\right)$

$\mathrm{S}_{\mathrm{j}+1}$ is the definitive prediction output. When $\mathrm{i}=1$, we predict based on all the previously occurred elements of the sequence. When $\mathrm{i}=\mathrm{j}$, we predict based on just the next element (e.g. Markovian processes).

Sequence classification is another example of sequence prediction. It includes the prediction of a class label for a specified input sequence. For example, DNA (deoxyribonucleic acid) sequence classification falls into this category. Given a DNA sequence of A, C, G, T (where each letter represents one of the four basic constituent molecules collectively known as nucleotides: cytosine $(\mathrm{C})$, guanine $(\mathrm{G})$, adenine $(\mathrm{A})$, and thymine $(\mathrm{T})$ ) values, predict whether the sequence codes for a coding or non-coding 
region [2], [3]. A genomic DNA segment, such as AGTACGTCCGATGACT, is a string of amino acids without any temporal connotation attached to their order in the sequence. Fig. 1 shows an example of how genomic sequencing can be used for gene prediction by Open Reading Frames (ORFs). Gene prediction is the process of determining where a coding gene might be in a genomic sequence. The length of functional proteins is from where DNA transcription begins (i.e., Start codon), to where it ends (Stop codon). Therefore, a functional protein is searchable by codons start and stop points in a DNA sequence. This is important in gene prediction because it can reveal where coding genes are in an entire genomic sequence. In this example, a functional protein can be discovered using ORF3 because it begins with a Start codon, has multiple amino acids, and then ends with a Stop codon, all within the same reading frame.

There are two steps to make a sequence prediction:

Step 1) Training sequences are the task of training of a sequence prediction model by using some previously seen sequences. For instance, a sequence prediction model can be trained for machine operational and control sensors data for the prediction of a specific type of defect in the manufacturing plant.

Step 2) use the trained sequence prediction model to predict the new sequences (i.e., predict the next element of a new sequence). For example, using the trained defect prediction model to predict the upcoming defects.

Interpreting and relating the sequence of events that occur in a production process allows formulating the causal relationship between events. The expectation of this activity is to create a genetic construction of the process and is the main contribution of this research work. Such a construction is analogous to medicine (i.e., good gene causes healthy outcomes, and bad gene causes illness). It provides a perspective to predict the outcome of a process (e.g., end product Defect/Pass) based on the genetic constellation of the process, as the contiguous, i.e., events chain up during the process life cycle. The genetic register of the process will be saved in a gene/DNA of process library, where the "good genes" (optimum solution creating events) and "bad genes" (fail conducing events) and the sequence of their occurrences will be registered and used for optimization purposes by encouraging good genes and eliminating bad genes. In the latter case, by adjusting machines, material, logic etc., to prevent/avoid the occurrence of bad genes (e.g. defect inducing events).

For ease of understanding and to explain event-based process sequencing, the proposed GIP concept borrows some of the classical terms and descriptors of genetics and genome sequencing from biology science.

The proposed GIP registering technique is tested and validated through an industrial case study for quality assessment in PCB fabrication and assembly manufacturing. The creation of production genomics helps
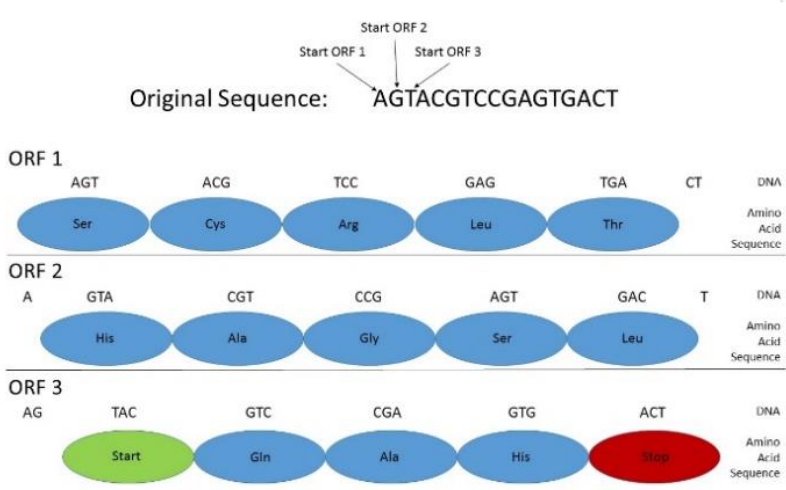

Fig. 1. An example of Gene prediction (Borrowed from [4])

in the prediction of defects and prevention through rectification of the defect, causing genes, leading to a ZeroDefect manufacturing process. To the best knowledge of the authors, such an approach to in-process Quality Control and Management is a step change compared with classical online-offline and post-process quality analysis.

In the following sections, a review of the most relevant sequence learning methods available in the literature is presented, followed by a detailed description of the proposed method and its application in an industrial case study. For performance comparison purpose, the same experiment is conducted by GIP and some other Neural Network (NN) methods for defect prediction. The comparison helps the authors to explain the merits and applicability of the proposed method for problem-solving in real-world industrial applications.

\section{RELATED WORKS}

Sequence learning's problems complexity and diversity are so varying that a single approach could be able to suffice to master the field. In the literature, there are many different applications for sequential learning, from the prediction scheme in navigation [5] to pattern recognition and prediction in [6]-[8].

Depending on the nature of the system and type of data, several methods have been proposed and applied. In this section, several relevant methods to the proposed technique will be reviewed.

Machine learning techniques examine the pattern of seen data and generate rules to discover recurring patterns. Machine learning algorithms learn from a sequence of events and 'experiences' with respect to a class of tasks [9]. The concept of machine learning is, therefore, the study of approaches and methods which can be applied to generalization and learning observed/seen instances pattern and construct rules in order to make a prediction when faced with new instances [10]-[13].

A taxonomy of machine learning methods is presented in fig. 2 [14]. Different machine learning methods have different approaches to learn sequences patterns. In the continue, some methods which use Temporal Sequence Learning (TSL) will be explained briefly. 


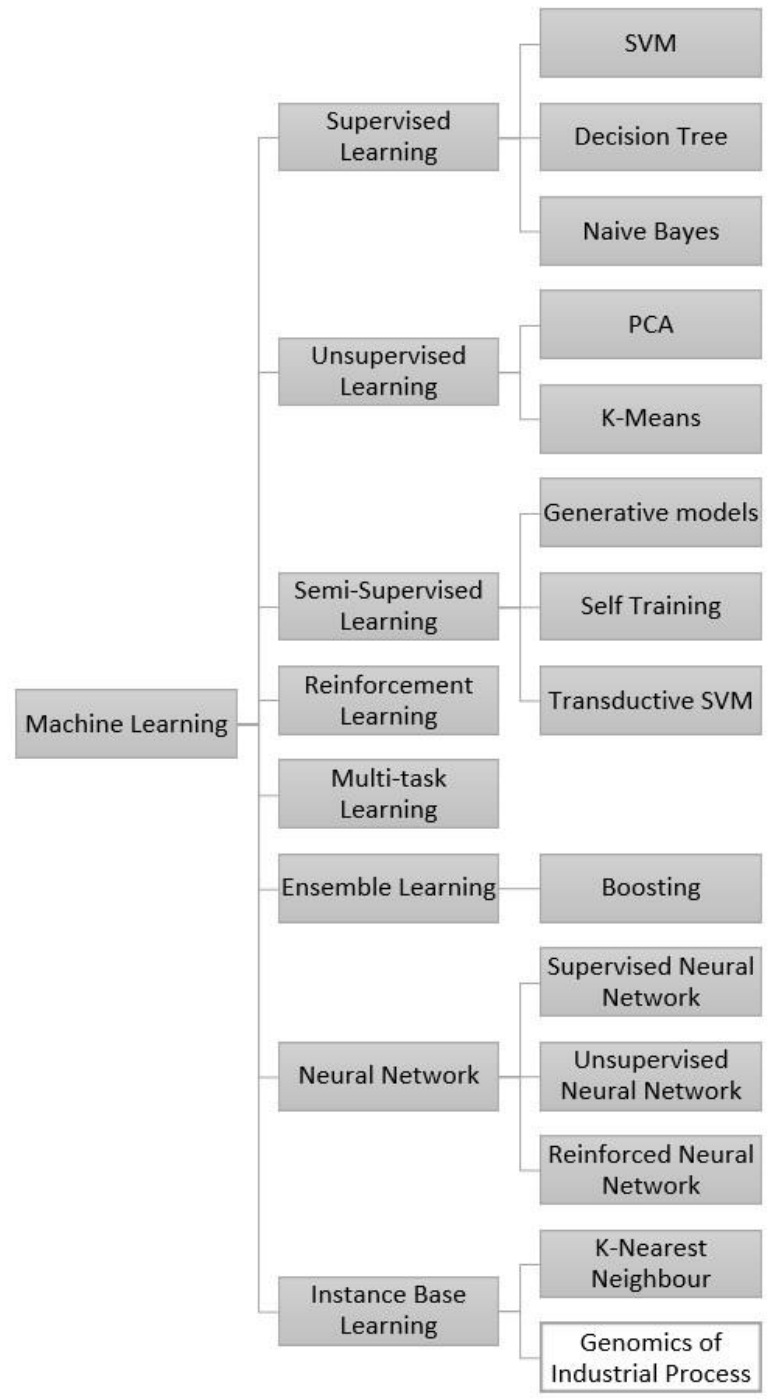

Fig. 2. Types of Learning

Hidden Markov Models (HMM) is a method deployed for sequence learning (including both generation and recognition). This model learns fundamental state transition probability distributions from observed data. The sequence generation can apparently be managed by this type of model. A comparison of supervised and unsupervised learning approaches of HMMs has been reviewed in [15]. After a theoretical comparison of both methods, a controlled experiment compares results obtained. The outcomes present that supervised learning methods performance is poor because they impose binding conditions in terms of data labelling, involves applicants' biases, calculate unreliable results due to the absence of constructs an efficient Maps with higher performance, and fewer applicants intervene. Temporal-Difference [16] (used mainly in reinforcement learning) and NN learning methods [17] are other examples of sequential learning of temporal patterns.

A time-series represents a sequence of acquired values that are measured through time span. Such series includes an ordered sequence of observations of finite sequence length, which are often taken through time span or space.
For decades, time-series have been applied in prediction and forecasting applications and theories. However, mostly, they rely on mathematical equations, simulation and/or learning techniques to represent the evolution of time series data. The body of literature in time-series is devoted to time-series classification applications in machine learning and a wide range of industries [18]- [23].

One popular TSL based machine-learning technique is instance-based learning (IBL) [24]. In this technique, an instance dictionary stores the set of representative instances. New unseen instances would be classified according to their relationship to previous seen stored instances. k-NN is an example of the instance selection methods which were developed based on instance-based learning algorithms. $\mathrm{k}-\mathrm{NN}$ is a typical example of classification methods, in which a new instance is assigned the label of many of the k dictionary instances according to domain-specific "closest" or "similarity" measurement. In temporal sequence domains, the similarity or closest measure is often taken to be the Euclidean distance [25]. This classification method is between top-performing methods. However, the complexity of IBS increases by growing the volume of data. A distinctive problem in k-NN is deciding which instances should be stored for generalization. Storing too many instances require large memory space and slow computation execution speed [26].

An evolutionary instance selection algorithm for IBL introduced by De Haro-García \& et al. in [27]. Although evolutionary algorithms are efficient in performance, a pitfall of them is the essence of storing all training instances subsets in memory space for large datasets, which might also impact the efficiency of the testing task. A solution to this problem introduced in [28] with an instance selection method that removes redundant and noisy instances. [29] provides a survey of existing techniques used to reduce storage requirements in instance-based learning algorithms, including different reduction and/or reconstruction algorithms of the training set. However, such storage reduction advantage usually becomes less interesting when we know it compensates with accuracy reduction. One of the recurrent problems with these instance selection methods is that although significant reductions in storage requirements are obtained, this reduction often comes at the cost of degraded accuracy.

IBL algorithms have a wide range of applications, from stock market prediction [30] to anomaly (Outlier) detection [31]. In anomaly-detection tasks, the anomalies can be formulated from learning of behaviours of the system in terms of temporal sequences of data. Chandola \& et al. [32] present an approach to cast the anomaly-detection task in an IBL framework. Their approach is to transform the temporal unordered sequence of observations into metric time-spaces via a similarity/closest measure that includes their intra-attribute dependencies.

With the growing availability of streaming online/realtime data, there is a significant demand for online/real-time 
sequence learning algorithms. With a focus on TSL terminology and IBL methods' performance and storage requirement problems, in the next section, an online/realtime sequence learning algorithm named Event Sequence Prediction (ESP) technique with a novel storage reduction method is introduced. This sequence learning algorithm is more efficient in that it requires much smaller computing and storage resources. The proposed ESP technique learns the event of complex sequencing in real-time while existing types of learning (such as statistical and machine learning) methods are not well suited to solve such real and practical needs and desires from industrial applications.

\section{SEQUential EVEnT PREDICTION_ THE THEORY OF EVENT-BASE GENOMICS OF INDUSTRIAL PROCESS (GIP)}

The Microelectronic fabrication and assembly process at times creates up to $50 \%$ defective products that need to be thrown away. Classical quality control techniques mainly rely on post-process quality assessment and statistical process control methods. Experts will then try to relate the patterns of quality loss to machine and material states at the time of production. Such techniques have limited impact and have proven obsolete with modern manufacturing processes. The intention here is to develop an accurate and applicable quality assessment and process correction system that could adjust the production system so that during the process, potentials of quality losses are identified in real-time, system is alerted, and corrective measures taken, leading to the optimal prize of zero-defect manufacturing (EU H2020 FoF research and innovation program under grant agreement No 723906).

As reviewed in the related research and literature section, all current data-driven and learning algorithms that could meet such complex demand were not available. All learning-based methods require training and large sets of data that neither exists about the raw material feed, the state of the machine and the type of defects generated. Furthermore, no formal and verifiable data/knowledge exists of the correlations between systems parameters that relate to quality loss (defect analytics).

The challenge was to develop and upgrade the real-time data acquisition on the production process and find a method of automatically interlinking the causal relationships between raw material state as well as machine state and capability of meeting product design specifications. This is especially timely since, in industrial applications, quick understanding of the state of the system (input parameters of system) and taking action means savings and improvement in quality, productivity, energy efficiency and sustainability performance (latent system outputs). Therefore, the closer the analysis and action is to the live operations, the more useful they will be in practice. Furthermore, the application should be easy to implement and follow by industrial controllers, operators, and decision-makers. The proposed method introduces a general framework for using Event-Clustering technique [37] in ESP. It has been named as a theory of event-based genealogy of process since this prediction method borrows the terminology used in genetics (in general terms) and similarly labels process events. Akin to DNA chains, the string of events are symbolized representing manufacturing process causal relationships and contiguous occurrences.

Analogous to the formation of polypeptides chains that manifest themselves as functions of life at the molecular level, each chain of signals in an industrial system registers function in a process. The chains present the linear sequence of events where a specific constellation can be interpreted as the building blocks of a process. For example, the chain of events that forms the DNA of process that successfully produces a "good product" or otherwise. The sequence of events that normally leave a traceable signature produces a registrable gene. Some constellations of genes (DNA) will lead to "good" products, and some lead to defects. Identifying such defect generating events will eventually lead to detection and prediction of the causes of a defect in a process (output). By identifying the defect generating genes in the process, methods for detecting them and countering (control reactions) or remedial actions (change of pathway) will lead to reduction and elimination of defect and waste in industrial systems. In this paper, an example of manufacturing where the genome of defect type $\mathrm{x}$ is presented as a sequence (or chain) of events (genes alphabetically labelled) that shifts state of the system to the generation of defect $\mathrm{x}$ is discussed. Defect $\mathrm{x}$, for example, can be defined as a combination of repeatable events labelled $\mathrm{A}$ to $\mathrm{D}$ (alphanumerically assigned), and defect $\mathrm{x}$ would happen if a sequence of ABBCD occurs. A, B... are representative of distinguish system states which will be explained in detail in the next sections. Noteworthy, the application of this method is not limited to quality control and could be used for other purposes such as process control (stabilization) application. i.e. instead of registration of bad genes that lead to defects, good genes which make the system stable in an optimized or desired point through registered scenarios/sequences can be registered.

What distinguishes the proposed ESP techniques from other sequence learning techniques is its simplicity and the speed at which it extracts and learns all existing sequential learning as well as its storage reduction technique and then processes the necessary information in near real-time. There is no reliance on a set of predefined rules such as good or bad genes or time-consuming investigation on the patterns. More importantly, unlike heuristic methods, the proposed technique does not rely on any prejudgment of the data relevancy, that is, normally a characteristic of expert interference and in that respect, it is an unbiased method.

\section{A. The Theorem of GIP}

The assumptions and basic parameters of the proposed EventTracker and EventiC such as Discrete Event System (DES), Tigger Data (TD) and Event Data (ED), Trigger and 
Event Threshold (TT, ET) are presented in [33], [34]. Further parameters of the proposed sequencing method are as the following:

\section{- Sequence}

A Sequence is expressed as the value or state of the actual I/O data at a given time instance. This data can be expressed in a binary, integer, or decimal format. For instance,

$$
\left(\mathrm{I}_{1}, \mathrm{I}_{2} \ldots \mathrm{I}_{\mathrm{m}}\right) \rightarrow \mathrm{f}_{\mathrm{t}}\left(\mathrm{O}_{1} \ldots, \mathrm{O}_{\mathrm{n}}\right)
$$

$\mathrm{f}_{\mathrm{t}}\left(\mathrm{O}_{1} \ldots, \mathrm{O}_{\mathrm{n}}\right)$ represents the value of outputs at a specified time instance.

- Genome of Industrial Process (GIP)

A GIP is the sequence of events that lead to a definitive output(s). The definitive outputs are observable/measurable states (e.g. defect type $\mathrm{x}$ ) called a genome (process) of defect type $\mathrm{x}$ formation.

\section{- $\quad$ Length of GIP}

The length of GIP is the number of elements (i.e. genes) in a sequence. At present, determining the length of genomes are specified by the definition of process (processing steps) or expert knowledge. Trial and error in the period of learning can also be an alternative.

\section{B. Sequence Database}

All labelled genomes are stored in a database called a lookup table. These genomes are labelled, timestamped, and contain a definition. This database is continuously updated with new events and repeated events; thus, the gene pool will be identified after a period of observing the system. For each type of industry, such genes become unique or shared. Thus, the family trees of processes, machines, tools, and networks could emerge.

\section{Sequence-generating rules, Genealogy of Process}

A set of rules and protocols govern the constraints and objectives of the system. For example, a definitive output or inputs boundaries and constraints could be defined by these rules and with respect to the nature of the system studied. A genealogy of process will emerge. With the advent of machine networks and large-scale monitoring and integrated systems, the necessary data for this process will exist. For example, a specific machine that produces micro semiconductors will have a pedigree with shared genes with the same brand, and more importantly, with other brands, such interrelationships will build the family trees, where behaviours and patterns can be extracted for better control logic. New generations of machines/processes can be created by eliminating fault generating machines leading to evolution and optimization of the design.

\section{Sample scan rate}

Sample scan rate is a specified time interval where the duration can potentially range from microseconds (high frequency) to hours (low frequency) and is chosen by the system expert based on the nature of the application. For example, applications such as safety systems require shorter scan intervals between events, whilst other applications such as wastewater plant process require longer intervals [34].

\section{E. ESP Algorithm.}

The proposed ESP ranks and groups the relationship between system input and output parameters. A combination of event tracking [34] and Rank Order Cluster (ROC) [33] groups and assigns weights to the system input against system outputs. The array of inputs and their weight vis-à-vis system outputs are used in generating the event sequences genomes and their likelihood of occurrence. In the following, a step-by-step implementation of the method is presented.

\section{1) The implementation of the EventiC algorithm}

The ESP algorithm begins with dimensionality reduction through a rank order clustering technique. Event clustering (EventiC) sensitivity analysis helps to find a correlation between inputs and output and then calculate the weight of impact of each input on the output parameters. It runs Rank Order Clustering (ROC) (See in Fig. 3) to identify the group of most relevant inputs and outputs.

\section{2) Definition of Rules and definitive output( $s$ )}

The system inputs boundaries and definitive output(s) are defined by a set of rules. The system inputs boundary and constraint help the outliers, such as noise removed from the dataset. Moreover, the rules set includes the definitive system outputs. The definitive outputs are the range of outputs we are searching for as the objective of our prediction. For example, if the aim is to predict defects by the ESP algorithm, the definitive system output(s) is the specific type of defects and their properties.

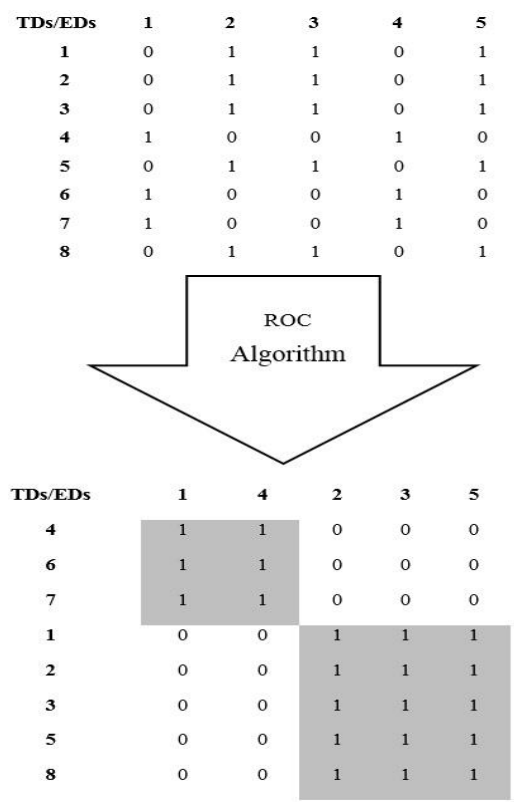

Fig. 3. Input triggered/Event Data incidence matrix before (up) and after (down) implementation of ROC algorithm 
3) Genomes labelling and sequential event differentiation

The next step in the ESP algorithm is the labelling of the events. According to the definitions, states (e.g. defect) detection is conducted. Then based on chosen genome length, all contiguous sequences of events prior to the defect state are labelled.

An example will demonstrate how patterns are discovered patterns in event sequences. Fig. 4 illustrates the labelling and sequence differentiation process. Here, the system observes the definitive output(s) at $\mathrm{T}=4$. Then the value of relevant sequences will be read and labelled in the following time sequences: $\mathrm{t}_{0}, \mathrm{t}_{1}, \mathrm{t}_{2}, \mathrm{t}_{3}, \mathrm{t}_{4}$.

The first element in the sequence is registered at $t_{0}$ and labelled as ' $A$ '. According to the EventiC output and TD/ED, the second sequence labelled could be a new state differentiable to the prior state, only if the system state's difference passes the threshold, then a new label is assigned to the new state as ' $\mathrm{B}$ ' this is continued until the moment of state $\mathrm{E}$ which is the occurrence of the defect.

Subsequently, the formation of events from $t_{0} \ldots t_{4}$, five consecutive events form the genome strand 'ABCDE'. Note that in this example, only four prior events are considered sufficient to form the genome of defect formation.

\section{4) Training and storing the genomes}

At the end of the training period, all trained genomes/scenarios will be stored in the sequence database as a lookup table. In real-time prediction, these stored genomes will be used for the prediction of the system trained definitive outputs(s) and declaring the likelihood of occurrence. In the above example, if during prediction, sequence ' $A$ ' is detected, the likelihood of defect will be $20 \%$ ( 1 out of five possible events. Consequently, if ' $\mathrm{A}$ ' $\rightarrow$ ' $\mathrm{B}$ ' $\rightarrow$ ' $\mathrm{C}$ ' $\rightarrow$ ' $\mathrm{D}$ ' occurred in the real-time prediction, more likely the next sequence is ' $\mathrm{E}$ ' unless proven otherwise. However, if state ' $E$ ' did not occur, and ' $F$ ' occurs, the algorithm has detected two different genomes for a definitive output, and the genome length must be extended to six to generate new labels and genome.

\section{$F$. Level of confidence for ESP results}

The length of a GIP needs to be extended if the GIP prediction leads to two different definitive outputs. The

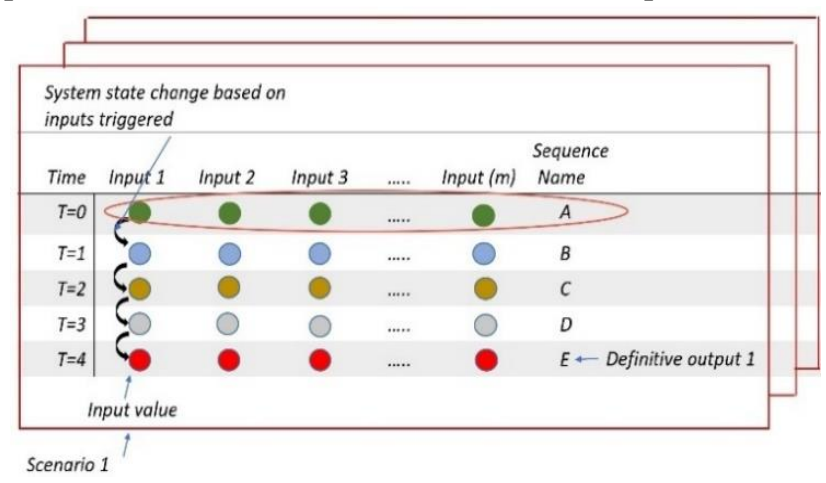

Fig. 4. Genome labelling and sequential differentiation example length would be extended until the GIP meet a unique definitive output.

A case study is presented in the following section to further explain the GIP implementation in a real industrial case. Here every step of the process is registered in the form of a chain of events coded in the form of a sequence of genomes representing the DNA of process scenarios that lead to "good" or "defect" outcomes.

\section{THE DNA OF MICRO SEMICONDUCTOR MANUFACTURING PROCESS}

A defect-prone process of a printed circuit board (PCB) fabrication and assembly is presented. The assembly process requires consists of dispensing conductive glue on a Liquid Crystal Polymer (LCP) substrate with tight design specifications; the microelectronic components need to be precisely positioned on the wafer using a machine. The process involves the placement and adhesion of die/components the size of 800um $x$ 900um) with conductive paste into a laser-cut cavity. The assembly process is undertaken on a mother panel containing 18 individual circuits each circuit containing 20 components. Prior to the deployment of the proposed GIP, the placement machine did not have any online optical inspection capability and no method to automatically correct the amount of glue dispensed in real-time into the cavity. Consequently, a visual volumetric inspection of the assemblies (post-process quality control) took place after batches of products were made; subsequently, an analysis of machine and raw material states were made, and adjustments were made to the machine retrospectively at a scheduled maintenance period. Note that the old practice produced a significant number of defects between 5 to $50 \%$. As the design becomes more complex, the defects increase exponentially. The glue dot dispensed volumes require to be controlled; otherwise, it might lead to some severe defects. The possible defects are as the following:

a) Excess of glue_ leak up the excess of glue to the side of the die causing solder shorts - action: reject to scrap

b) Insufficient glue_it causes component adhesion issues action: rework/scrap

The actual single glue dot is shown in fig. 5. There is a need to be able to improve this process and ideally control the amount of glue being dispensed into the cavity automatically and during the production process. As a first step introducing an online automated optical inspection machine would help to provide the necessary data for analysis and trend learning.

\section{A. The Rheological behaviour of Glue}

To define output system parameters (observational and modelled), characterization of the time-dependent rheological behaviour of glue is conducted in [35]. With air compressibility and liquid inertia consideration, an inferential model of the dynamics of the flow rate of the 


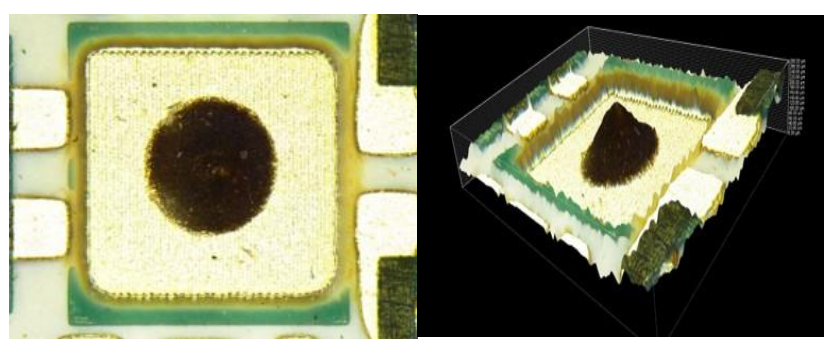

Fig. 5. A single glue dot 2D (left) and 3D (right) images

dispensed liquid is sensitive to the air volume in the syringe (i.e., the definitive output). An experiment emulating the machine glue dispensing was conducted in [36]. Given various glue characteristics, needle conditions, operational and environmental conditions, the experiments were repeated. Various control mechanisms were deployed to capture and reduce inconsistency (e.g., defining the range of glue dispensing patterns; thus, the operational ideal (good drops) and non-ideal drops (defective) were extracted.

A schematic diagram of the system is shown in fig. 6 . This is a schematic of a conventional time-pressure dispensing process. An air supply is utilized to give pressurized air in a blend with a valve to control the term of the pressurized air. Through a transmission line, pressurized air is connected to a syringe. In addition, the syringe pushes the liquid out of the needle. Once the liquid is released from the needle, it drops onto a board, then it streams or spreads on the board to the point that a balanced profile is shaped. The needle is operational typically for 30000 to 60000 and lasts 14 days.

The relationship between the amounts of dispensed glue, glue level in the syringe, applied pressure, glue temperature and machine depression (The needle age) have been taken into consideration. This model shows a high sensitivity of the amount of glue dispensed to applied pressure and glue temperature, and the model can predict the amount of glue dispensed.

\section{B. Measuring the volume of glue dot}

Optical inspection is a non-destructive technique apply in the electronic industry, including PCB defect detection. A comprehensive literature review in [37] appraises the techniques used to detect defective PCBs. The review also analyses the inspection algorithms used for detection and interpretation of defects in the electronic components. The

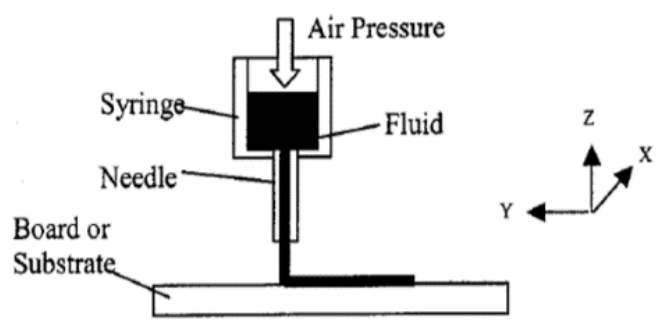

Fig. 6. Time-pressure dispensing schematic [39] algorithms include data pre-processing, feature extraction and classification.

Excess and Insufficient glue on the PCB joints are two types of defects that may occur during the manufacturing and assembly process. Electric tests and human visual inspection tests are common methods of detection and interpretation, but novel automated optical inspection technologies alongside modern inspection algorithms for PCB quality control are gaining impetus [38] [39].

The detection and defect interpretation method in this article deploys a high-resolution laser scanner that scans the areas of interest on a PCB and extracts the geometric information in terms of three-dimensional (3-D) point clouds. Moreover, a Regression-Net (RNet), a 3D Convolutional Neural Network (3DCNN) framework, is used to estimate the volume of the glue on the designated die attachment on PCBs [40]. In this study, two well-known deep learning methods of VoxNet [41] and PointNet [42] have been adjusted to the volume estimation with their final classification layer and subnetwork replacement with a fully connected layer NN. In the end, the applied RNet model accurately predicts the volume of glue deposits both before and after die attachment with $92 \%$ accuracy. The proposed system uses a custom scanning component that extracts a high-resolution point cloud of a glue deposit or, more interestingly after die attachment when only a small part of glue is visible from the glue fillet that is formed around each die.

\section{Dispensed machine data parameters and corresponding glue volume}

In this experiment, the dispensing machine dispenses 500 glue dots on 10 LCP substrates in 2.5 second intervals. Date about machine state and the ambient conditions during the dispensing were collected from installed operational sensors (Temperature sensor (tempSen), Air pressure sensor (supplyAps), Vacuum sensor (HouseVa), Dispensed voltage (DispensedVolt) and Syringe pressure sensor (Syringe Psi)) on the dispensing machine and log to a repository to be stored (fig. 7). The operation data-sampling rate of the system is set at $500 \mathrm{~ms}$. This implies that the machine state is registered at $500 \mathrm{~ms}$ intervals; this allows a 5 -event registration prior to the next glue drop. The five contiguous system states could be a good indicator of GIP length. Appendix I and II (fig. 12-13) present all the 500 glue samples of 10 LCPs glue dots and corresponding machine operational and environmental sensor parameters.

\section{Sequential event-modelling algorithm training (Scenarios of Process Genomes)}

The step-by-step implementation of the proposed ESP is as follows:

\section{1) Event-Clustering sensitivity analysis algorithm}

The sequential event-modelling algorithm begins with dimensionality reduction through the rank order clustering 
technique. EventiC [33] helps to find the many-to-many correlation between inputs and output parameters reveals the sensitivity of the glue shape (output) against the machine and ambient state parameters (system input).

The interpretation of the data series is based on triggers and events. Only those fluctuations in the data series that are interpreted as triggers represent state change. Falsenegative and positive tests were conducted, as explained in [33], and the trigger threshold was set at 5\%. It means that any alteration in input sensors (dispense machine operation data) and glue volume, more than 5\% has been considered as a new event. The EventiC results are shown in Table I.

These results show Syringe pressure has a high degree of impact on the glue volume while 'HouseVa' has a medium impact, and these two sensors should be considered as important sensors in the sequential algorithm. The remaining sensors have a low degree of impact on the glue volume. The low-ranking parameters could be ignored in the next steps (dimension reduction).

\section{2) Defect detection}

The second step in the sequential event modelling algorithm is to ascertain the acceptable range for glue volume. The minimum and maximum satisfactory ranges for glue volume are 0.04 and $0.15 \mathrm{~mm}^{3}$, respectively. Any larger (excess of glue) or smaller (Insufficient glue) glue has been defined as a defect. In this experiment, as seen in Appendix I, three insufficient glue types (dispense numbers of 40, 209 and 445) and two excess glue type (dispense numbers of 21 and 210). The data associated with each event represents the qualia of the system state for that specific time. The labels are alphanumerically assigned (e.g., A-Z). For example, in this experiment, defective drops are labelled as C, D, and G. The reason for three different labels is that they have distinctive defect types as far as glue dispensing is concerned (fig. 8).

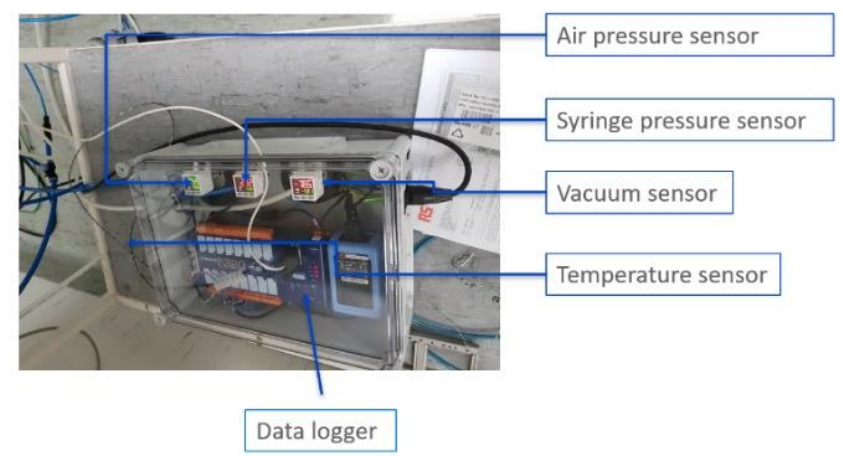

Fig. 7. Sensors and data logger installed on the dispensing machine

Table I. EventiC output between the dispensing machine sensors and glue volume

\begin{tabular}{|c|c|}
\hline Input/output & Glue Volume \\
\hline SyringePsi & $\mathbf{7 9 \%}$ \\
\hline HouseVa & $\mathbf{5 7 \%}$ \\
\hline DispensedVolt & $42 \%$ \\
\hline TempSen & $38 \%$ \\
\hline SupplyAPsi & $29 \%$ \\
\hline
\end{tabular}

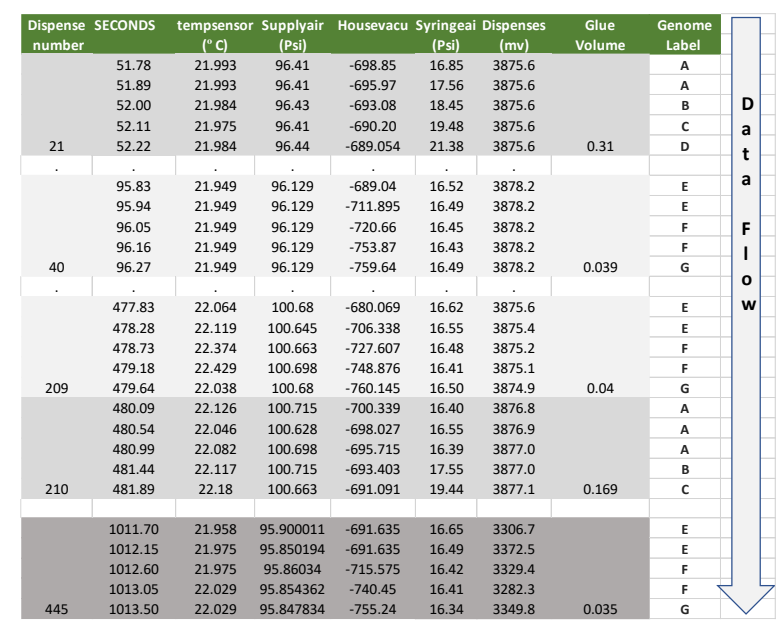

Fig 8. ESP training

3) Genomes labelling and sequential differentiation

In the training process, a series of 4 events of machine state is labelled, the fifth registered event is the glue state (good/defective). Labelling begins with genome length selection. The default genome length is set at five since there are five operational data samples between two consecutive glue dispenses. As the glue state is determined (fifth event), the previous four machine events are labelled. Each detected machine state is compared with previous labelled events. If the difference is more than $5 \%$, a new event and label is assigned; otherwise, it will be considered as no new state is detected and the same event added to the chain.

For this specific example, the values of 'SyringePsi' and 'HouseVa' input parameters determine the state change of the machine. Fig. 8 illustrates the results of running the algorithm for 500 glue dots (10 LCPs), but only five instances of the defect are displayed here. The first trained

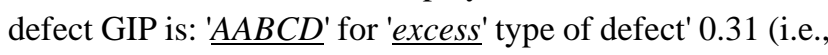
defect type D). There is also another GIP for defect type C, (i.e., excess of glue ' 0.15 '), which is' $\underline{A A B C}$ '. For the 'insufficient' type of defect, the GIP is 'EEFFG'. Defect type $\mathrm{G}$ occurred twice in this example. The results of gene definition, labels, and their sequence of occurrence (DNA) are stored in the process genes pool for the specified process (See fig. 9).

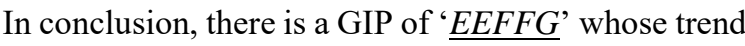
is to lower bound defect (insufficient glue volume) and were repeated two times in the training dataset and a GIP of ' $A A A B C$ ' with a trend to upper bound defect (excess of glue volume). The ESP algorithm will be able to predict the dispensing in real-time for both types of defects through sequence differentiation and cross-check with the database of Gene labels dictionary and defects GIP list.

\section{ESP PERFORMANCE COMPARED TO OTHER ML ALGORITHMS.}

The performance of the prediction of ESP is compared with the three most popular data mining and ML [43] methods. Random Forest Regression (RF), k Nearest 

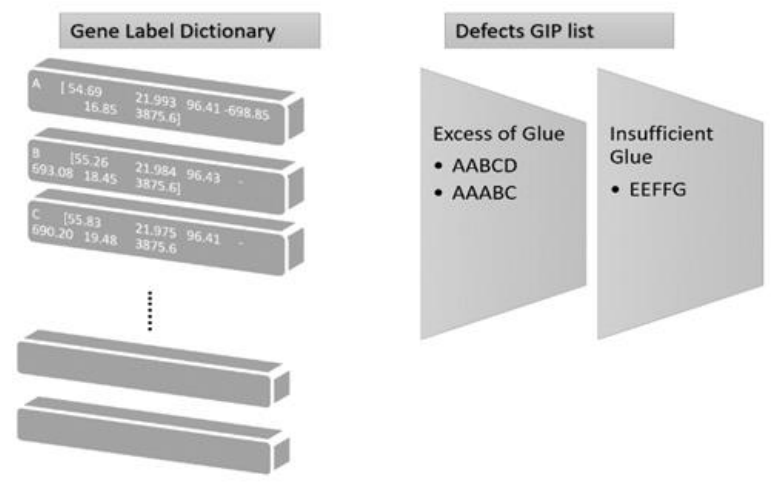

Fig. 9. A database of Gene labels dictionary and defects GIP pool. Neighbours (kNN) and Multi-Layer Perceptron (MLP) network models were chosen due to their high accuracy and application in similar industrial applications.

RF Regression is one of the most effective and conventional ML models for predictive analytics. The purpose of the algorithm is to ascertain the output from multiple decision trees instead of relying on individual decision trees [44], [45]. Hyperparameters are associated with an RF regression model and used to optimize its performance and include the number of decision trees (n_estimators) and the number of features considered by each tree when splitting a node (max_feature). Hyperparameters optimization is performed by doing an exhaustive search over selected parameter values. In this paper, we have set 50 for n_estimator and 20 for max_feature.

MLP network is one of the most popular and practical architectures of Artificial Neural Networks (ANN). In MLP, every single neuron is connected to its contiguous neuron, with varying impact weights that represent the relativity of the different neuron inputs to the others. The overall weights of the inputs are shifted to the hidden neurons, where it is transformed using an activation function. The other neurons use the outputs of these hidden neurons as inputs, in turn, where they pass through another transformation [46]. The architecture of the MLP that has been employed in this paper is based on two hidden layers with 8 and 10 neurons, respectively. In this model, the weights and biases are modified using the Adaptive Moment Estimation (Adam) optimization function. Moreover, the learning rate was set to 0.01 .

To evaluate and compare the performance of the selected methods with the proposed ESP, the same 500 experimental datasets were deployed for the analysis. $90 \%$ of the dataset has been selected as training data, assuming at least a defect from each type in the training dataset. And the remaining $10 \%$ of the data (i.e., 50 samples) were treated as testing data (unknown or unseen data). Fig. 10 represents the model training and prediction procedure.

Mean absolute error (MAE) and mean squared error (MSE) between the observed and predicted results were used to compare the results. Table II summarises the outcome of the tests after 10 replications. The results show both types of errors in RF model are less than $\mathrm{kNN}$ and MLP models in this experiment. Then in the continuation, RF method is selected to be compared with the proposed ESP. However, even with RF regression method, the predictive model will not be able to predict all defects since there are limited observable defects samples in the training dataset (See the RF model results for sample 35 in fig.11). One of the pitfalls of classical ML models is their need for large sample sizes (i.e., observable defects) to build an accurate inferential prediction model. Table III presents the number of errors of both RF and ESP methods in the training dataset.

The comparison between the proposed ESP and RF predictive methods shows that RF method can only reduce uncertainty if the number of observations is statically acceptable. Since the number of observed defects normally in modern industrial processes is very low, such technique falls short and, with the associated uncertainty, does not provide strong decision support. In contrast, due to the nature of observation and detection of causal relations, The ESP methods do not suffer from uncertainty. The proposed ESP builds a lookup dictionary of genomes based on the occurred defects and corresponding genomes. The proposed

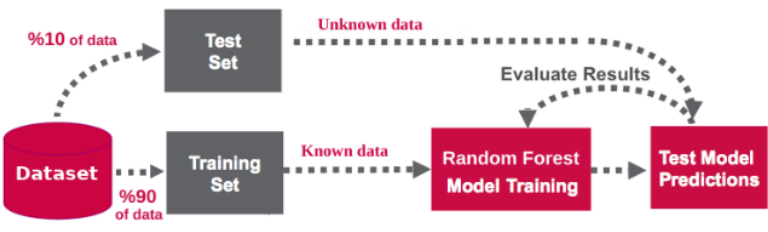

Fig. 10. Model training and predictions

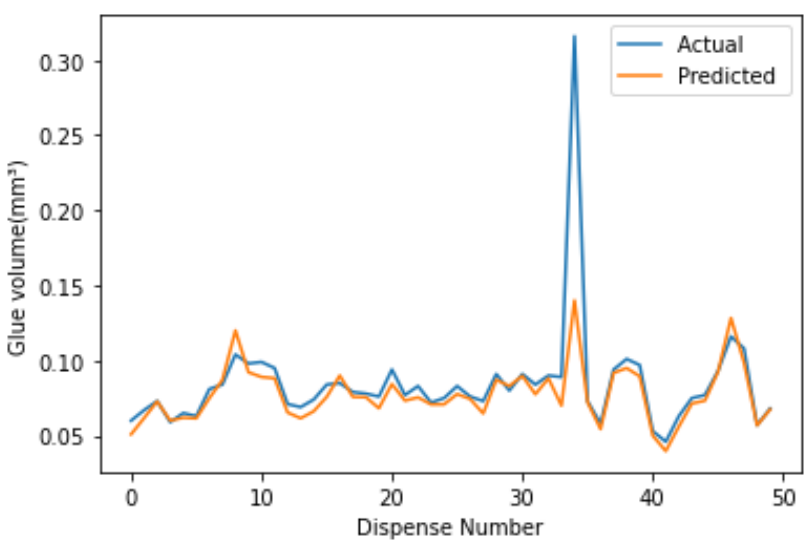

Fig.11. Predicted data (Red) through the RF model and actual data (Blue) in the test dataset

Table II. MAE and MSE between the actual and predicted results after ten times repetition

\begin{tabular}{|c|c|c|c|}
\hline & RF & kNN & MLP \\
\hline MAE & 0.00084 & 0.00256 & 0.006541 \\
\hline MSE & $7.436861 \mathrm{e}-06$ & $3.24511 \mathrm{e}-05$ & 0.00018 \\
\hline
\end{tabular}

Table III. Number of errors in prediction

\begin{tabular}{|c|c|}
\hline Method & Number of errors \\
\hline RF & $1(100 \%)$ \\
\hline ESP & 0 \\
\hline
\end{tabular}


ESP method is also superior to similar lookup table solutions used in failure prediction [47] because implementing the EventiC method (dimension reduction) reduces memory usage significantly compared to other lookup table methods. The second advantage of the ESP method is its real-time self-training algorithm. This selftraining feature, in comparison to $\mathrm{NN}$ offline timeconsuming training algorithm, is beneficial.

\section{CONCLUSION AND FUTURE WORKS}

This paper describes the practical implications of utilizing modern data acquisition techniques, real-time data analytics and learning methods in industrial environments. The challenges posed by the complexity and timing demand of the case study motivated us to go beyond the existing ML and AI techniques that could not offer satisfactory results on their own. The new technique for real-time sequencing strategy not only has the capability to predict events for key performance indicators of a given industrial system but also provides an alternative state-space description of industrial processes in the identification of root causes of suboptimal performances. A comparison of the proposed ESP method and the RF machine learning method is conducted in their performance and level of confidence. The results have helped increase confidence that by deploying ML and AI in Manufacturing/Industrial processes, there is a possibility to build Zero Defects - Zero Waste processes.

The proposed Theory of Process GIP takes its inspiration from biology and genetic science, thus creating a process for labelling and establishing sequential event differentiation from observed information. These labelled genomes of the process are being used to predict the events according to their occurrence sequence. GIP can be classified as Granular computing ( $\mathrm{GrC}$ ) technique. $\mathrm{GrC}$ is an approach for reasoning a system and dividing information into smaller pieces (Genes) to see if they differ on a granular (trigger threshold) level. This approach could become a new tool for deep and reinforcement learning. A future pathway for research is to explore its ability to rationalize the existing imbalanced data labelling during training datasets by reducing reliance on statistical means. This problem is prevalent in manufacturing environments where potentially a mixture of weighted factors could cause serious misinterpretation and loss of productivity.

One of the applications of GIP to be explored is in the enhancement of learning and reasoning capabilities of Cognitive Digital Twins (CDTs). The gene pool created by GIP real-time gene recognition throughout the learning process allows the nature of the physical process to be transformed into reusable and referenceable knowledge. The gene sequencing (DNA of the process) can be a powerful tool for interpreting and demonstrating the knowledge graphs in CDT. Another potential area to explore is the role of GIP in simplifying and relaxing rulebased models (e.g. [48],[49]).

\section{ACKNOWLEDGEMENT}

This project has received funding from the European Union's Horizon 2020 research and innovation program under grant agreement No 723906.

\section{REFERENCES}

[1] Sun, R., \& Giles, C. L. (Eds.). (2003). Sequence learning: Paradigms, algorithms, and applications. Springer.

[2] Nguyen, N. G., Tran, V. A., Ngo, D. L., Phan, D., Lumbanraja, F. R. Faisal, M. R., ... \& Satou, K. (2016). DNA sequence classification by convolutional neural network. Journal of Biomedical Science and Engineering, 9(05), 280.

[3] Zhou, C., Cule, B., \& Goethals, B. (2016). Pattern-based sequence classification. IEEE Transactions on Knowledge and Data Engineering, 28(5), 1285-1298.

[4] Pandakekok, Gene Prediction.3 December 2016. [Online]. Available https://commons.wikimedia.org/wiki/File:Gene_Prediction.png\#file history.

[5] Yin, J., Wang, N., \& Perakis, A. N. (2017). A real-time sequential ship roll prediction scheme based on adaptive sliding data window. IEEE Transactions on Systems, Man, and Cybernetics: Systems, (99), 1-11

[6] Gonzalez, C. C., \& Burke, M. R. (2018). Motor Sequence Learning in the Brain: The Long and Short of It. Neuroscience, 389, 85-98.

[7] Crivelli-Decker, J., Hsieh, L. T., Clarke, A., \& Ranganath, C. (2018). Theta oscillations promote temporal sequence learning Neurobiology of learning and memory, 153, 92-103.

[8] Baker, R., Dexter, M., Hardwicke, T. E., Goldstone, A., \& Kourtzi, Z. (2014). Learning to predict: Exposure to temporal sequences facilitates prediction of future events. Vision Research, 99, 124-133.

[9] Karl Hansson, Siril Yella, Mark Dougherty, Hasan Fleyeh," Machine Learning Algorithms in Heavy Process Manufacturing", American Journal of Intelligent Systems 2016, 6(1): 1-13

[10] Simeone, O. (2018). A very brief introduction to machine learning with applications to communication systems. IEEE Transactions on Cognitive Communications and Networking, 4(4), 648-664

[11] Das, S., Dey, A., Pal, A., \& Roy, N. (2015). Applications of artificial intelligence in machine learning: review and prospect. International Journal of Computer Applications, 115(9).

[12] Y. Liu, J. Bi, Z. Fan, Multi-class sentiment classification: the experimental comparisons of feature selection and machine learning algorithms, Expert Syst. Appl. 80 (2017) 323-339 www.scopus.com

[13] Dey, A. (2016). Machine learning algorithms: a review. International Journal of Computer Science and Information Technologies, 7(3), 1174-1179.

[14] Simeone, O. (2018). A very brief introduction to machine learning with applications to communication systems. IEEE Transactions on Cognitive Communications and Networking, 4(4), 648-664.

[15] Khodabandelou, G., Hug, C., Deneckere, R., \& Salinesi, C. (2014) Supervised vs. unsupervised learning for intentional process model discovery. In Enterprise, Business-Process and Information Systems Modeling (pp. 215-229). Springer, Berlin, Heidelberg.

[16] Wörgötter, F., \& Porr, B. (2005). Temporal sequence learning, prediction, and control: a review of different models and their relation to biological mechanisms. Neural computation, 17(2), 245319.

[17] Sutskever, I., Vinyals, O., \& Le, Q. V. (2014). Sequence to sequence learning with neural networks. In Advances in neural information processing systems .pp. 3104-3112.

[18] George, K., \& Mutalik, P. (2017). A multiple model approach to time-series prediction using an online sequential learning algorithm. IEEE Transactions on Systems, Man, and Cybernetics: Systems, 49(5), 976-990.

[19] Cho, S., \& Foroosh, H. (2018, March). A Temporal Sequence Learning for Action Recognition and Prediction. In 2018 IEEE 
Winter Conference on Applications of Computer Vision (WACV) (pp. 352-361). IEEE.

[20] Abanda, A., Mori, U., \& Lozano, J. A. (2019). A review on distancebased time series classification. Data Mining and Knowledge Discovery, 33(2), 378-412.

[21] Wang, H., Zhang, Q., Wu, J., Pan, S., \& Chen, Y. (2019). Time series feature learning with labelled and unlabeled data. Pattern Recognition, 89, 55-66.

[22] Nóbrega, J. P., \& Oliveira, A. L. (2019). A sequential learning method with Kalman filter and extreme learning machine for regression and time series forecasting. Neurocomputing.

[23] Abanda, A., Mori, U., \& Lozano, J. A. (2019). A review on distancebased time series classification. Data Mining and Knowledge Discovery, 33(2), 378-412.

[24] Y. Liu, J. Bi, Z. Fan, Multi-class sentiment classification: the experimental comparisons of feature selection and machine learning algorithms, Expert Syst. Appl. 80 (2017) 323-339.

[25] Zhang, X., Li, Y., Kotagiri, R., Wu, L., Tari, Z., \& Cheriet, M. (2017). KRNN: k Rare-class Nearest Neighbour classification. Pattern Recognition, 62, 33-44.

[26] Zhang, S., Li, X., Zong, M., Zhu, X., \& Wang, R. (2018). Efficient knn classification with different numbers of nearest neighbors. IEEE transactions on neural networks and learning systems, 29(5), 17741785.

[27] de Haro-García, A., Pérez-Rodríguez, J., \& García-Pedrajas, N. (2018). Combining three strategies for evolutionary instance selection for instance-based learning. Swarm and Evolutionary Computation, 42, 160-172.

[28] Krawczyk, B., Triguero, I., García, S., Woźniak, M., \& Herrera, F. (2019). Instance reduction for one-class classification. Knowledge and Information Systems, 59(3), 601-628.

[29] Gong, C., Liu, T., Tang, Y., Yang, J., Yang, J., \& Tao, D. (2018). A regularization approach for instance-based superset label learning. IEEE Transactions on Cybernetics, 48(3), 967-978.

[30] Y. Chen, Y. Hao, A feature weighted support vector machine and knearest neighbor algorithm for stock market indices prediction, Expert Syst. Appl. 80 (2017) 340-355.

[31] Ren, H., Ye, Z., \& Li, Z. (2017). Anomaly detection based on a dynamic Markov model. Information Sciences, 411, 52-65.

[32] Chandola, V., Banerjee, A., \& Kumar, V. (2012). Anomaly detection for discrete sequences: A survey. IEEE transactions on knowledge and data engineering, 24(5), 823-839.

[33] Danishvar, M., Mousavi, A., \& Broomhead, P. (2018). EventiC: A real-time unbiased event-based learning technique for complex systems. IEEE Transactions on Systems, Man, and Cybernetics: Systems, (99), 1-14.

[34] S. Tavakoli, A. Mousavi, P.Broomhead, "Event Tracking for RealTime Unaware Sensitivity Analysis (EventTracker)," IEEE Trans. Knowledge and Data Engineering, vol.25, no.2, pp.348-359, 2013.

[35] IZADPANAH MEHRKISH, N. (2017). The mathematical model of glue dispersion and amelioration its performance in presence of optimal controllers. MSc Dissertation. Brunel University.

[36] Chen, X. B., Schoenau, G., \& Zhang, W. J. (2001). Dynamic modeling of the flow rate in time-pressure fluid dispensing processes. In Proceedings of IPACK (Vol. 1).

[37] Abd Al Rahman, M., \& Mousavi, A. (2020). A Review and Analysis of Automatic Optical Inspection and Quality Monitoring Methods in Electronics Industry. IEEE Access.

[38] T. Vafeiadis, N. Dimitriou, D. Ioannidis, T. Wotherspoon, G. Tinker, and D. Tzovaras, "A framework for inspection of dies attachment on PCB utilizing machine learning techniques,” J. Manage. Anal., vol. 5, no. 2, pp. 81-94, Apr. 2018.

[39] Fonseka, C. L. S. C., \& Jayasinghe, J. A. K. S. (2018). Feature extraction and template matching algorithm classification for PCB fiducial verification. J. Achievements Mater. Manuf. Eng., 86(1), 1432 .
[40] N. Dimitriou, L. Leontaris, T. Vafeiadis, D. Ioannidis, T Wotherspoon, G. Tinker, D. Tzovaras, Fault diagnosis in microelectronics attachment via deep learning analysis of $3 \mathrm{~d}$ laser scans, IEEE Transactions on Industrial Electronics To appear. doi:10.1109/TIE.2019.2931220.

[41] Maturana and S. Scherer, "VoxNet: A 3d convolutional neural network for real-time object recognition," in Proc. IEEE/RSJ Int Conf. Intell. Robots Syst., 2015, pp. 922-928.

[42] R. Q. Charles, H. Su, M. Kaichun, and L. J. Guibas, "PointNet: Deep learning on point sets for 3d classification and segmentation," in Proc. IEEE Conf. Comput. Vis. Pattern Recognit., 2017, pp. 77-85.

[43] Settouti, N., Bechar, M. E. A., \& Chikh, M. A. (2016). Statistical comparisons of the top 10 algorithms in data mining for classification task. International Journal of Interactive Multimedia and Artificial Intelligence, 4(1), 46-51.

[44] Ahmad,T., Chen,H. (February 2019). "Nonlinear autoregressive and random forest approaches to forecasting electricity load for utility energy management systems," Sustainable Cities and Society, Volume 45, , PP. 460-473.

[45] Cui, Y., Ahmad, S., \& Hawkins, J. (2016). Continuous online sequence learning with an unsupervised neural network model. Neural computation, 28(11), 2474-2504.

[46] Heidari, E., Sobati, M. A., \& Movahedirad, S. (2016). Accurate prediction of nanofluid viscosity using a multilayer perceptron artificial neural network (MLP-ANN). Chemometrics and intelligent laboratory systems, 155, 73-85.

[47] Kim, Y. S. (2016). Failure Prediction of Metal Oxide Varistor Using Nonlinear Surge Look-up Table Based on Experimental Data Transactions on Electrical and Electronic Materials, 16(6), 317-322.

[48] Huang Z, Li M, Mousavi A, Danishvar, M. and Wang, Z. (2019), EGEP: An Event Tracker Enhanced Gene Expression Programming for Data Driven System Engineering Problems, IEEE Transactions on Emerging Topics in Computational Intelligence, 3(2), 117 - 126.

[49] Razgon, M., and Mousavi A. (2020), Relaxed Rule-based Learning for Automated Predictive Maintenance: proof of concept, Algorithms, 13(9)

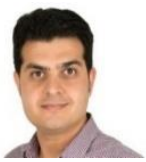

Morad Danishvar is a research scientist in the System Engineering Research Group at Brunel University London, UK. He received his $\mathrm{PhD}$ degree from Brunel University London in 2015. His research interest is in Data Science, Data analytics and Machine Learning, AI, Digital Manufacturing, DSS, Real-Time Systems Modelling and Optimization. Morad also is deputy director at Brunel University’s Digital Manufacturing Centre (DMC).

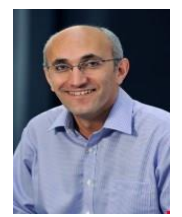

Alireza Mousavi is a Reader at College of Engineering, Design and Physical Sciences, Brunel University. His research interest is in smart supervisory control and data acquisition systems applied to real-time systems modelling and optimization. The key areas of application are stochastic modelling, ontology alignment and sensor networks. He is a senior member of IET and IEEE.

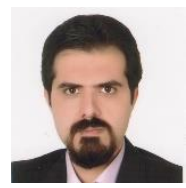

Sebelan Danishvar received his $\mathrm{PhD}$ degree from Tarbiat Modares University, Tehran, Iran, in 2007, in Electrical and computer engineering. His current research activities are modelling and simulation, machine learning and image processing. He currently collaborates and works on EU Horizon 2020 Z-Factor and HISTEED projects. 


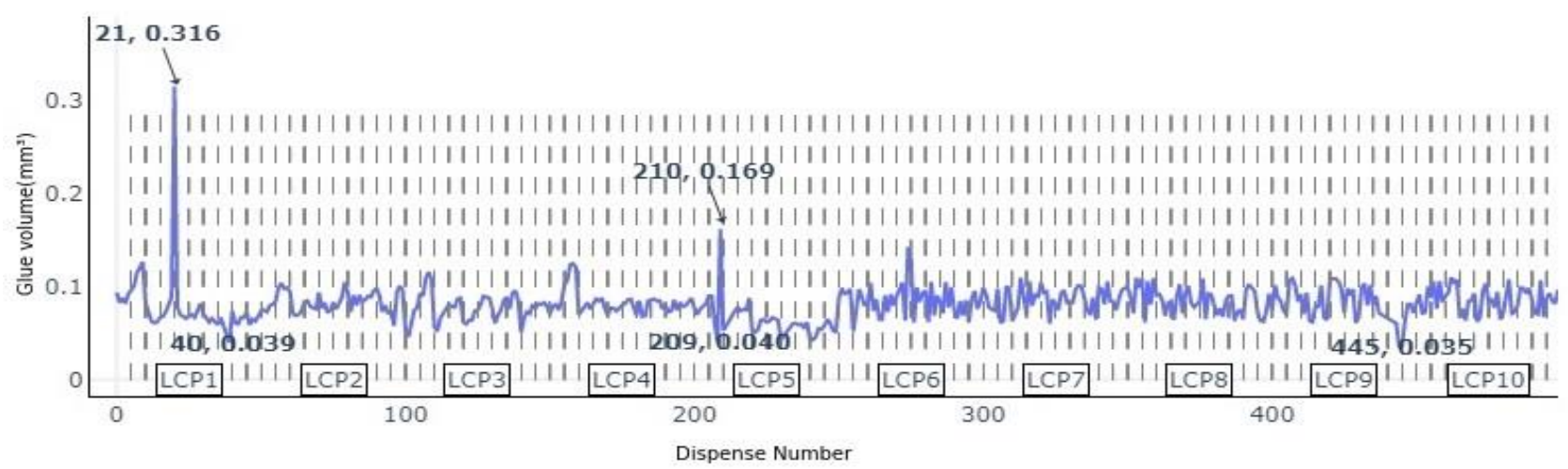

Fig. 12. 500 Dispensed glue volumes profile

APPENDIX II. Dispense machine operational and environmental sensor parameters.
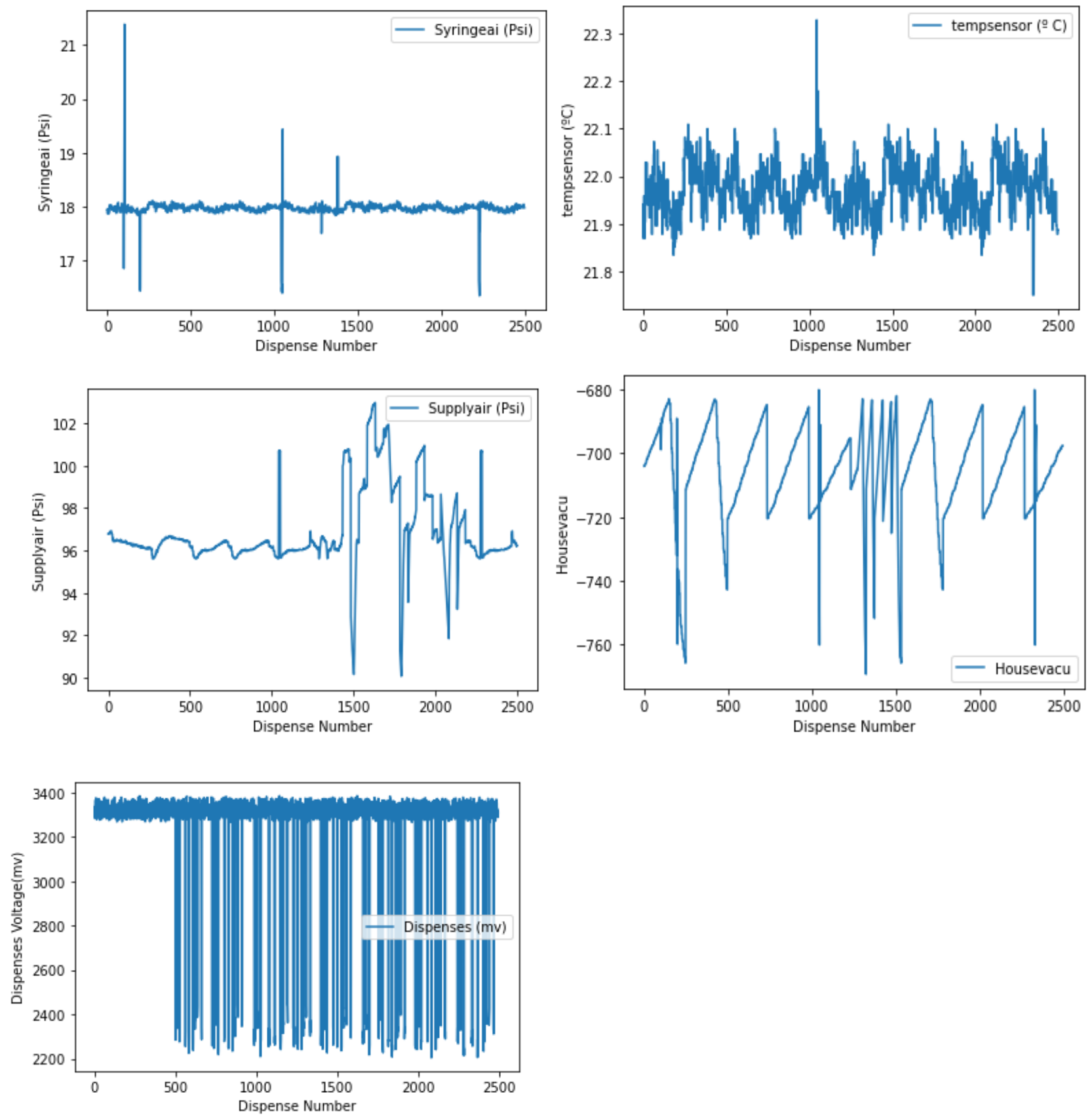

Fig. 13. Dispense machine operational and environmental sensor parameters 